\title{
Photobiomodulation Therapy Prevents Dysgeusia Chemotherapy-Induced in Breast Cancer Women Treated with Doxorubicin Plus Cyclophosphamide: A Triple-Blinded, Randomized, Placebo-Controlled Clinical Trial
}

Cássia Emanuella Nóbrega, Malta

Federal University of Ceara: Universidade Federal do Ceara

Anna Clara Aragão Matos Carlos

Unichristus: Centro Universitario Christus

Manuele Carine Maciel de Alencar

Cancer Institute of Ceara: Instituto do Cancer do Ceara

Eveline Fernandes Alves e Silva

Cancer Institute of Ceara: Instituto do Cancer do Ceara

Victor Bruno Caitano Nogueira

Cancer Institute of Ceara: Instituto do Cancer do Ceara

Ana Paula Negreiros Nunes Alves

UFC: Universidade Federal do Ceara

Fábio Figueiredo Chaves

Cancer Institute of Ceara: Instituto do Cancer do Ceara

José Fernando Bastos de Moura

Cancer Institute of Ceara: Instituto do Cancer do Ceara

Paulo Goberlânio Barros Silva ( $\sim$ paulo_goberlanio@yahoo.com.br)

Federal University of Ceara: Universidade Federal do Ceara https://orcid.org/0000-0002-1513-9027

Research Article

Keywords: Dysgeugia, Low Intensity Light Therapy, Anthracyclines, Cyclophosphamide, Breast Neoplasm.

Posted Date: August 20th, 2021

DOl: https://doi.org/10.21203/rs.3.rs-687053/v1

License: (c) (1) This work is licensed under a Creative Commons Attribution 4.0 International License.

Read Full License 


\section{Abstract \\ Purpose}

To evaluate the effectiveness of photobiomodulation (PBMT) in preventing dysgeusia in breast cancer patients treated with doxorubicin-cyclophosphamide (AC).

\section{Methods}

This is a phase II, randomized, triple-blind, placebo-controlled clinical trial involving 112 breast cancer patients treated with AC. The patients were divided equally into two groups: a test group treated with $2 \mathrm{~J}$ red laser and $3 \mathrm{~J}$ infrared laser on 21 points that were symmetrically distributed on the tongue on day 0 of four cycles of AC, and an equal placebo group treated with simulated PBMT to blind the patient, evaluator, and statistician. The clinicopathological and sociodemographic data, results of the hematological tests, taste test, and subjective taste analysis, and the QoL, ECOG performance status, body mass index, and other side effects were recorded. The data were analyzed using ANOVARM/Bonferroni, Friedman/Dunn, and chi-square/Fisher's exact tests.

\section{Results}

PBMT patients showed less objective and subjective taste loss $(p<0.05)$. On the other hand, the placebo group showed a higher ECOG status $(p=0.037)$ and more significant weight loss $(p<0.001)$ after four cycles of AC. The QoL was significantly higherin the PBMT group $(p<0.05)$ at all assessment periods, and PBMT treatment also reduced the incidence of cachexia $(p=0.020)$, anorexia $(p<0.001)$, diarrhea $(p$ $=0.040)$, oral mucositis $(p=0.020)$, and vomiting $(p=0.008)$.

\section{Conclusion}

PBMT reduced the taste loss and improved the overall health status and QoL of patients with breast cancer treated with AC.

\section{Trial registration}

: Brazilian Clinical Trials Registry (www.ensaiosclinicos.gov.br) approval numberRBR-9qnm34y, registered on $01 / 05 / 2021$

\section{Introduction}


Chemotherapy for breast cancer generally combines antineoplastic drugs, orally or intravenously, that inhibit tumor growth and normal fast-growing cells $[1,2,3]$. Its use in breast cancer can occur after (adjuvant chemotherapy) or before (neoadjuvant chemotherapy) the surgical procedure, during the maintenance phase, or even palliatively in patients with metastatic tumors [2-4].

Although highly effective for most breast tumors, doxorubicin,in combination with cyclophosphamide (AC), has limiting factors such as cardiotoxicity and emergence of multiple drug resistance.

Antineoplastic agents used in chemotherapeutic treatment are toxic to normal fast-growing tissues, with the manifestation of side effects during follow-up. The effects predominate in cells with rapid cell division, such as those of the hematopoietic tissue, germ tissue, hair follicle, gastrointestinal lining, and oral cavity [1-3].

Among the various side effects in women treated with $\mathrm{AC}$, the second most frequent non-hematologic complications is taste loss (97.3\%) [1, 3, 5]. Taste is essential for recognizing flavors and textures of substances [6]. The disruption of taste tissue homeostasis can be harmful to the taste system [6-7], and chemotherapy can cause direct damage to taste bud cells or indirectly alter brain regions responsible for taste perception [5-8]. Patients with dysgeusia presented with worse overall quality of life (QoL) and reduced food intake and cachexia [6-8].

Photobiomodulation (PBMT) has been used to promote photochemical changes in target tissues without structural loss $[9,10]$. These changes occur due to the absorption by the target tissue of the light emitted by PBMT, which occurs at the cellular level, improving metabolism, cell proliferation, and maturation, and decreasing inflammatory mediators. Because it stimulates mitochondrial activity, PBMT acts as an antiinflammatory, analgesic, and wound-healing agent [10-12].

Considering that there are no treatment/prevention protocols for chemotherapy-induced dysgeusia, and that PBMT is a low-cost therapeutic modality that has enormous benefits in controlling other side effects in the mouth such as oral mucositis, this study aimed to evaluate the influence of PBMT in preventing dysgeusia in breast cancer patients treated with AC.

\section{Materials And Methods}

\section{Study design and ethical considerations}

This is a phase II, randomized, triple-blind, placebo-controlled clinical trial registered in the Brazilian Clinical Trials Registry (www.ensaiosclinicos.gov.br) following the CONSORT guidelines for clinical trials, with approval protocol number RBR-9qnm34y and ethics approval number 3.286 .363 (Instituto do Câncer do Ceará, ICC).

\section{Participants and clinical setting: inclusion, exclusion, and withdrawal criteria}

Patients aged $>18$ years, with stage II-IV breast cancer, and free of previous chemotherapy were selected for the first adjuvant, neoadjuvant, or palliative treatment with drug protocols of the combined 
doxorubicin (Adrimicin ${ }^{\circledR}$ ) and cyclophosphamide (Cytoxan $\left.{ }^{\circledR}\right)(A C)$. Chemotherapeutic protocols cannot be associated with other drugs.

Patients with a history of radiotherapy to the head and neck, who smoke, with anemia, with untreated diabetes mellitus, with a history of drug use that significantly altered the salivary flow, saliva composition, or taste [6,7], or who are taking centrally acting analgesics or anxiolytics and antidepressants were excluded from the study. Study participants were also excluded if they dropped out of treatment or the study, required a change of chemotherapy protocol that will replace the AC protocol, discontinued chemotherapy for any reason, developed extreme toxicity, or died (Figure 1).

All patients were treated at the chemotherapy outpatient clinic of the Haroldo Juaçaba Hospital/ICC from July-2019 to January-2020.

\section{Study groups and experimental protocol}

After signing the informed consent form we performed a baseline clinic evaluation and QoL assessment. So, laser therapy was performed. A therapy EC laser model (DMC®, São Carlos, SP, Brazil) with $100 \mathrm{~mW}$ of light output power at a continuous wavelength of $660 \pm 10 \mathrm{~nm}$ (red) and $808 \pm 10 \mathrm{~nm}$ (infrared) was used. The device has a tip with an area of $0.09842 \mathrm{~cm}^{2}$, which was kept in light contact with the treated area during the protocol applications. Patients were treated on the day of chemotherapy infusion and on day 0 (D0) and D+21 (end of one cycle and start of another), from the beginning of the first chemotherapy cycle for four cycles $(\mathrm{C} 1, \mathrm{C} 2, \mathrm{C} 3$, and $\mathrm{C} 4)$.

Then, $2 \mathrm{~J}$ of red-light per dot, which is used for tissue regeneration during chemotherapy [11], and $3 \mathrm{~J}$ of infrared light per dot, which is used for paresthesia [13] (energy density $=20.32 \mathrm{~J} / \mathrm{cm}^{2}$ and $30.48 \mathrm{~J} / \mathrm{cm}^{2}$, respectively) were applied in fourteen points spread symmetrically across the dorsum of the tongue $[10,14]$ (Supplementary File 1).

\section{Outcomes and data analysis}

\section{Clinicopathological and sociodemographic data collection and oral health profile}

We evaluated the patients' medical records and collected the clinicopathological and sociodemographic data, including age, tumor location, comorbidities, drugs in use, menarche, and menopause, parity, nutritional support, psychological support, and previous surgical and/or radiotherapy treatments. Tumor stage, histological type, and immunohistochemistry profile (hormonal receptors, HER-2 and ki-67) and sociodemographic data were also recorded.

Prior to the first chemotherapy session, the patient's oral cavity was inspected to measure the index of decayed, missing, and filled teeth (DMFT) and the degree of tooth mobility [15]. The collection and evaluation of non-stimulated salivary secretion were performed using the expectoration method at each chemotherapy [16]. 


\section{Taste acuity evaluation}

\section{Taste test}

The objective taste test was performed before applying the low-intensity laser on the days of chemotherapy administration. Taste thresholds were performed using $0.01 \mathrm{~mol} / \mathrm{L}, 0.032 \mathrm{~mol} / \mathrm{L}, 0.1$ $\mathrm{mol} / \mathrm{L}, 0.32 \mathrm{~mol} / \mathrm{L}$, and $1.0 \mathrm{~mol} / \mathrm{L}(0.01-1.0 \mathrm{~mol} / \mathrm{L})$ of glucose (sweet), sodium chloride (salty), citric acid (sour), and urea (bitter). A drop for each concentration was placed at the central region of the tongue and swallowed by the patient, starting with the lowest concentration. Then, the individual evaluated the stimulus for $15 \mathrm{~s}$ to perceive and identify the flavor. If recognition or identification did not occur, the next concentration was applied. Between the different flavor modalities, the patients rinsed their mouth with distilled water [17].

To calculate the taste loss variation, the - $\log 10$ of the concentration of each taste perceived by the patient was calculated, ranging from no taste alteration $(-\log 10(0.01)=2)$, minimal taste alteration $(-\log 10(0.032)=1.5)$, moderate taste alteration $(-\log 10(0.1)=1)$, strong taste alteration $(-\log 10(0.32)=0.5)$, and severe taste alteration $(-\log 10(1)=0)$. The sum of taste loss scores adjusted by $-\log 10$, which ranges from 0 to 8 , was divided by 8 and multiplied by 100 to obtain the Taste Sensitivity Score (TSS, \%), according to the formula below:

$\operatorname{TSS}(\%)=\frac{[-\log 10(\text { sweet })]+[-\log 10(\text { salty })]+[-\log 10(\text { sour })]+[-\log 10(\text { bitter })]}{8} \times 100$

\section{Subjective taste analysis}

During the chemotherapy cycles, we questioned the patient about his subjective perception of taste using a Visual Analog Scale (VAS), ranging from 0 to 10, where 0 corresponds to no change and 10 corresponds to a maximum loss of taste. The Common Terminology Criteria for Adverse Events (CTCAE) v3.0 criteria for adverse events classifies the patient's taste as no change (0) to changes in both taste and diet (2) [18]; and the scale of Subjective Total Taste Acuity (STTA) is a total taste acuity scale that classifies the taste as same acuity as before treatment (0) to complete loss of taste acuity (4) [19]. These scales were completed along with VAS at each cycle (every 21 days) of chemotherapy before applying the PBMT/placebo.

\section{QoL analysis, health status and side effects}

The Oral Health Impact Profile (OHIP)-14 questionnaire was administered on D0 of each cycle from the beginning of $\mathrm{C} 1$. The OHIP-14 is a subjective indicator that measures disability (Disability), discomfort, and handicap (Handicap) attributed to the oral condition through self-assessment and their relationship to the QoL. It consists of 14 questions, a reduced version of the OHIP-49. It is also scored on a Likert-type scale from 1 (never) to 5 (always) [20]. OHIP-14 and incidence of gastrointestinal tract-related side effects 
(anorexia, diarrhea, nausea, oral mucositis, and vomiting) were also evaluated were reported at each chemotherapy cycle such as

On $\mathrm{C} 1$ and $\mathrm{C} 4$ the body mass index (BMI) was calculated and patients were scored in ECOG scale in a range from 0 to 5 [21].

\section{Sample Size Calculation}

Based on a case-control study, Sánchez-Lara et al. [22] observed that patients with cancer present greater taste distortion than those without cancer (10\% vs. 33.3\%); thus, 51 patients per study group (chi-square test) were necessary to obtain a sample that represents, with $80 \%$ power and $95 \%$ confidence, the alternative hypothesis of this study, as estimated using the Fleiss method with continuity correction foreseeing sample loss. Given the possibility of sample loss, $10 \%$ was added to the sample, totaling 56 patients per study group.

\section{Randomization and Blinding}

The patients were randomly divided into the control group and the test group. Randomization (simple) was performed by a collaborator using the "=randomize()" command of Microsoft Excel (Microsoft Corporation ${ }^{\circledR}$ ) by simple randomization in study groups $A$ and $B$. Then, the randomization numbers were printed on sealed envelopes to identify the group to which the patient belonged. They were opened only by the leading researcher at the time of treatment.

The leading researcher was aided by two collaborators (ACMC and MCMA), who were unaware of the group to which the patient belonged, to perform PBMT protocol and evaluations, thus making the study blind to the evaluators. In addition, the laser protocol was equally applied to both groups; however, the leading researcher simulated the application by turning the device on and off immediately [23], thus blinding the patients. The statistician (PGBS) and the evaluator (ACMC) supporting the work were also unaware of the group to which each patient belonged. Thus, only the principal investigator knew the patients' groups, thus blinding the patient, evaluator, and statistician, making the study triple blind.

\section{Statistical Analysis}

Clinical data were expressed as absolute and percentage frequencies and compared using Pearson's chisquare or Fisher's exact test. Data from taste tests and dysgeusia scores were expressed as mean and SD and compared using Mann-Whitney or Friedman/Dunn tests (nonparametric data) (SPSS v20.0, $p<0.05$ ).

\section{Sample size power}

Based on the TSS of breast cancer patients evaluated after four cycles of AC administration treated with PBMT (57.82 $\pm 21.49 \%)$ compared to patients treated with PBMT placebo (43.78 $\pm 23.16 \%)$, the sample of 56 patients per study group used in this study had a power of $91.4 \%$ in rejecting the null hypothesis (Student's t-test). 


\section{Results}

Sociodemographic and clinicopathological characterization of breast cancer patients treated with AC

A total of 56 patients in each group remained until the end of the study. There was no difference in the distribution of these variables between the PBMT and placebo groups (Table 1). 
Table 1

Sociodemographic and clinicopathological profile of breast cancer patients treated with Doxorubicin and cyclophosphamide and undergoing PBMT protocol for dysgeusia prevention.

Group

$\begin{array}{lll}\text { PBMT placebo } & \text { PBMT test } & \text { p-Value }\end{array}$

Age

Up to 45 years old

$19(33.9 \%)$

$16(28.6 \%)$

0.541

$>45$ years old

$37(66.1 \%)$

$40(71.4 \%)$

Race

White

$22(39.3 \%)$

$26(46.4 \%)$

0.445

Brown

$34(60.7 \%)$

30 (53.6\%)

Source

Metropolitan Area

$24(42.9 \%)$

19 (33.9\%)

0.331

Countryside

$32(57.1 \%)$

$37(66.1 \%)$

\section{Education}

Can read and write

$3(5.4 \%)$

$1(1.8 \%)$

0.627

Elementary School

$27(48.2 \%)$

$24(42.9 \%)$

High School

$13(23.2 \%)$

$14(25.0 \%)$

Higher Education

$13(23.2 \%)$

$17(30.4 \%)$

\section{Nutritional orientation}

No

$16(28.6 \%)$

$14(25.0 \%)$

0.670

Yes

$40(71.4 \%)$

$42(75.0 \%)$

Before

37 (92.5\%)

40 (95.2\%)

0.818

During

$1(2.5 \%)$

$1(2.4 \%)$

After

$2(5.0 \%)$

$1(2.4 \%)$

\section{Psychological support}

No

Yes
$13(23.2 \%)$

$43(76.8 \%)$
$18(32.1 \%)$

0.291 $38(67.9 \%)$

${ }^{\star} p<0.05$, Fisher's exact test or Pearson's chi-square test $(n, \%) . C T=$ chemotherapy; HR = hormonal receptor; * TNM staging (Tumor size $(T)$, Nodal involvement $(N)$ and Metastasis $(M)$ ); 


\begin{tabular}{|c|c|c|c|}
\hline & Group & & \\
\hline Before & $41(97.6 \%)$ & $38(97.4 \%)$ & 0.367 \\
\hline During & $1(2.4 \%)$ & $0(0.0 \%)$ & \\
\hline After & $0(0.0 \%)$ & $1(2.6 \%)$ & \\
\hline Menarche $<12$ years old & $25(46.3 \%)$ & $20(38.5 \%)$ & 0.415 \\
\hline Menopause & $34(60.7 \%)$ & $30(53.6 \%)$ & 0.445 \\
\hline Children & 47 (87.0\%) & $48(85.7 \%)$ & 0.840 \\
\hline \multicolumn{4}{|l|}{$\mathrm{T}$} \\
\hline 1 & $3(5.6 \%)$ & $7(13.0 \%)$ & 0.035 \\
\hline 2 & $16(29.6 \%)$ & $26(48.1 \%)^{*}$ & \\
\hline 3 & $25(46.3 \%)^{\star}$ & $12(22.2 \%)$ & \\
\hline 4 & $10(18.5 \%)$ & $9(16.7 \%)$ & \\
\hline \multicolumn{4}{|l|}{$\mathbf{N}$} \\
\hline 0 & $11(19.6 \%)$ & $20(36.4 \%)$ & 0.264 \\
\hline 1 & $27(48.2 \%)$ & $20(36.4 \%)$ & \\
\hline 2 & $15(26.8 \%)$ & $12(21.8 \%)$ & \\
\hline 3 & $3(5.4 \%)$ & $3(5.5 \%)$ & \\
\hline \multicolumn{4}{|l|}{ M } \\
\hline 0 & $41(82.0 \%)$ & $42(85.7 \%)$ & 0.616 \\
\hline 1 & $9(18.0 \%)$ & $7(14.3 \%)$ & \\
\hline \multicolumn{4}{|l|}{ Primary tumor } \\
\hline Unilateral & $54(96.4 \%)$ & $55(98.2 \%)$ & 0.558 \\
\hline Bilateral & $2(3.6 \%)$ & $1(1.8 \%)$ & \\
\hline \multicolumn{4}{|l|}{ Phenotype } \\
\hline Luminal A & $7(12.5 \%)$ & $6(10.7 \%)$ & 0.803 \\
\hline Luminal B & $19(33.9 \%)$ & $23(41.1 \%)$ & \\
\hline Hybrid Luminal & $11(19.6 \%)$ & $12(21.4 \%)$ & \\
\hline
\end{tabular}




\begin{tabular}{|c|c|c|c|}
\hline & Group & & \\
\hline HER2 & $3(5.4 \%)$ & $1(1.8 \%)$ & \\
\hline Triple negative & $16(28.6 \%)$ & $14(25.0 \%)$ & \\
\hline HR & $37(66.1 \%)$ & $41(73.2 \%)$ & 0.411 \\
\hline HER2 & $14(25.0 \%)$ & $13(23.2 \%)$ & 0.728 \\
\hline \multicolumn{4}{|l|}{ Intent CT } \\
\hline Neoadjuvance & $33(58.9 \%)$ & $26(46.4 \%)$ & 0.389 \\
\hline Adjuvance & $20(35.7 \%)$ & $25(44.6 \%)$ & \\
\hline Palliative & $3(5.4 \%)$ & $5(8.9 \%)$ & \\
\hline \multicolumn{4}{|l|}{ Type of surgery } \\
\hline Quadrantectomy & $24(58.5 \%)$ & $24(47.1 \%)$ & 0.273 \\
\hline Total mastectomy & $17(41.5 \%)$ & 27 (52.9\%) & \\
\hline
\end{tabular}

Most of the patients treated had tumors at the T2 or T3 stage, with nodal involvement and without distant metastases. Patients treated with placebo had significantly more T3 tumor than those treated with PBMT $(p=0.035)$. Only three patients had bilateral metasynchronous tumors, and most only had a tumor in one breast. The phenotype of most tumors was luminal B, with most patients positive for hormone receptors and negative for HER2. Neoadjuvant therapy was used in most patients in both the PBMT and placebo groups, and most patients were treated with mastectomy and axillary emptying in both groups. These variables showed no significant differences between the two groups (Table 1).

\section{General health status and weight loss in breast cancer patients treated with AC and given PBMT for prevention of chemotherapy-induced dysgeusia}

Only eight patients needed to undergo two more AC cycles, for a total of six cycles of AC, but he patients were evaluated only for the first four cycles to standardize the evaluations. Most patients started chemotherapy treatment with ECOG 0 in $\mathrm{C} 1$ and by the end of $\mathrm{C} 4$. There was no significant variation in the PBMT group $(p=0.208)$, while the placebo group showed a significant increase in patients with ECOG $>1(p=0.016)$. At the end of $C 4$, there were significantly more $E C O G>1$ in the placebo group than in the PBMT group $(p=0.037)($ Table 2$)$. 
Table 2

General health status at the beginning and end of treatment with Doxorubicin and cyclophosphamide in breast cancer patients undergoing PBMT protocol to prevent chemotherapy-induced dysgeusia.

Group

\begin{tabular}{llll} 
& PBMT placebo & PBMT test & p-Value \\
Need for CT postponement & $5(9.1 \%)$ & $3(5.8 \%)$ & $0.514^{\mathrm{a}}$ \\
\hline AC chemotherapy protocol & & & \\
\hline AC-4 & $51(90.1 \%)$ & $53(94.6 \%)$ & $0.616^{\mathrm{a}}$ \\
AC-6 & $5(8.9 \%)$ & $3(5.4 \%)$ & \\
\hline
\end{tabular}

\section{ECOG in C1}

$\begin{array}{llll}0 & 42(75.0 \%) & 47(83.9 \%) & 0.366^{\mathrm{a}} \\ 1 & 13(23.2 \%) & 9(16.1 \%) & \\ 2 & 1(1.8 \%) & 0(0.0 \%) \\ 3 & 0(0.0 \%) & 0(0.0 \%)\end{array}$

ECOG in C4

$\begin{array}{llll}0 & 27(48.2 \%) & 41(73.2 \%)^{*} & 0.037^{\mathrm{a}} \\ 1 & 22(39.3 \%)^{*} & 13(23.2 \%) & \\ 2 & 5(8.9 \%)^{*} & 2(3.6 \%) \\ 3 & 2(3.6 \%) & 0(0.0 \%)\end{array}$

$\operatorname{BMI}\left(\mathrm{kg} / \mathrm{m}^{2}\right)$

C1

C4

p-Value

Percentage change BMI

BMI Variation

$>5 \%$ weight loss

${ }^{*} \mathrm{p}<0,05$, ${ }^{a}$ Fisher's exact test or Pearson's chi-square test ( $\left.\mathrm{n}, \%\right)$; ${ }^{\mathrm{b}}$ Mann-Whitney test; ${ }^{\mathrm{C}}$ Wilcoxon test $($ mean $\pm S D)$.

** CT (Chemotherapy); AC (Doxorrucicin and Cyclophosphamide); BMI (Body Mass Index); C1 and C4 (Chemotherapy cycle); ECOG (General Health Scale for Cancer Patients) 


\begin{tabular}{|c|c|c|c|}
\hline \multicolumn{4}{|c|}{ Group } \\
\hline Between $<5 \%$ and $>5 \%$ of weight & $33(58.9 \%) \star$ & \multicolumn{2}{|l|}{$22(39.2 \%)$} \\
\hline$>5 \%$ weight gain & $12(21.4 \%)$ & \multicolumn{2}{|c|}{$26(46.4 \%)^{\star}$} \\
\hline \multicolumn{4}{|l|}{$\mathrm{BMI}$ in $\mathrm{C} 1$} \\
\hline$<18.5 \mathrm{~kg} / \mathrm{m}^{2}$ & $0(0.0 \%)$ & $0(0.0 \%)$ & \multirow[t]{4}{*}{$0.837^{a}$} \\
\hline $18.5-25 \mathrm{~kg} / \mathrm{m}^{2}$ & $14(25.0 \%)$ & $15(26.8 \%)$ & \\
\hline $25-30 \mathrm{~kg} / \mathrm{m}^{2}$ & $21(37.5 \%)$ & $23(41.1 \%)$ & \\
\hline$>30 \mathrm{~kg} / \mathrm{m}^{2}$ & $21(37.5 \%)$ & $18(32.1 \%)$ & \\
\hline \multicolumn{4}{|l|}{$\mathrm{BMI}$ in $\mathrm{C4}$} \\
\hline$<18.5 \mathrm{~kg} / \mathrm{m}^{2}$ & $2(3.6 \%)$ & $1(1.8 \%)$ & \multirow[t]{2}{*}{0.297} \\
\hline $18.5-25 \mathrm{~kg} / \mathrm{m}^{2}$ & $13(23.2 \%)$ & $814.3 \%)$ & \\
\hline $25-30 \mathrm{~kg} / \mathrm{m}^{2}$ & $16(28.6 \%)$ & $25(44.6 \%)$ & \\
\hline$>30 \mathrm{~kg} / \mathrm{m}^{2}$ & 25 (44.6\%) & $22(39.3 \%)$ & \\
\hline \multicolumn{4}{|c|}{ 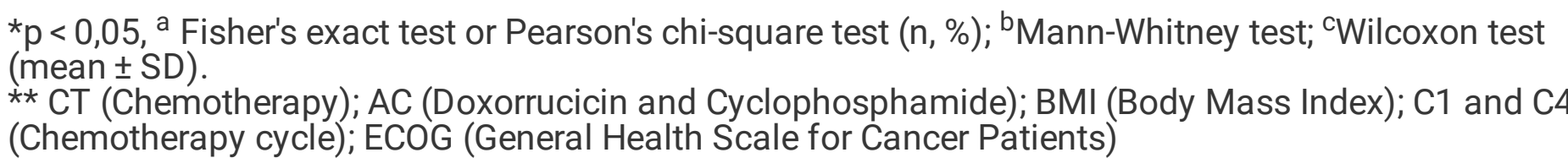 } \\
\hline
\end{tabular}

In $\mathrm{C} 1$, the patients' mean BMI started with no statistically significant difference between the two groups $(p=0.425)$ and remained to have no statistically significant difference at the end of $C 4(p=0.977)$. However, while the placebo group showed no significant change in BMI throughout the study $(p=0.560)$, patients in the PBMT group showed a significant $(p<0.001)$ increase of BMI $(p=0.006)$. Patients in the placebo group had a higher incidence of weight loss of $>5 \%$ or weight maintenance than patients in the PBMT group $(p=0.020)$. Nonetheless, there was no increase in the incidence of overweight/obesity in both groups (Table 2).

\section{PBMT prevents the depreciation of oral health and oral health-related QoL in breast cancer patients treated with $\mathrm{AC}$}

Patients in both the placebo group and PBMT group had a high DMFT at the beginning of the study ( $20.11 \pm 9.93$ and $20.55 \pm 10.07$, respectively), with no significant difference between the groups $(p=$ 0.726). There was no significant variation in the dose of $A C$ over time in either group, and there was no significant difference between the PBMT and placebo groups (Table 3 ). 
Table 3

Evaluation of Doxorubicin and Cyclophosphamide dose and oral health status over four chemotherapy sessions in breast cancer patients treated with PBMT protocol to prevent chemotherapy-induced dysgeusia.

\section{Cycle}

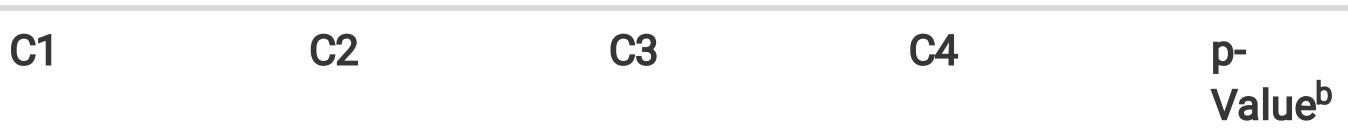

\section{Doxorubicin $\left(\mathrm{mg} / \mathrm{m}^{2}\right)$}

Placebo

$62.54 \pm 3.62$

$62.50 \pm 3.54$

$61.99 \pm 4.36$

$61.72 \pm 4.46$

$0.197^{b}$

Test

$62.62 \pm 2.74$

$61.15 \pm 9.11$

$60.63 \pm 9.30$

$60.24 \pm 9.45$

$0.090^{b}$

p-Value ${ }^{a}$

$0.922^{\mathrm{a}}$

$0.680^{\mathrm{a}}$

$0.735^{\mathrm{a}}$

$0.672^{\mathrm{a}}$

\section{Cyclophosphamide} $\left(\mathrm{mg} / \mathrm{m}^{2}\right)$

Placebo

Test

p-Value ${ }^{a}$

Salivary flow

Placebo

$0.91 \pm 0.37$

$0.93 \pm 0.38$

$0.89 \pm 0.34$

$0.88 \pm 0.35$

$0.892^{b}$

Test

$1.01 \pm 0.42$

$0.98 \pm 0.39$

$0.92 \pm 0.38$

$0.96 \pm 0.37$

$0.078^{b}$

$0.164^{a}$

$0.223^{a}$

$0.667^{a}$

$0.063^{\mathrm{a}}$

\section{Dental Mobility}

Placebo

$1.75 \pm 1.34$

$2.02 \pm 1.36$

$2.22 \pm 1.30$ *

$2.16 \pm 1.61^{*}$

$0.003^{b}$

Test

$2.05 \pm 1.33$

$2.14 \pm 1.31$

$2.12 \pm 1.36$

$2.06 \pm 1.39$

$0.534^{b}$

p-Value

\section{OHIP-14}

Placebo

Test

$0.655^{\mathrm{a}}$

$0.727^{a}$

$0.874^{a}$

$0.250^{\mathrm{a}}$

$9.09 \pm 6.30$

$12.75 \pm$

$7.27 *$

$13.09 \pm$
$7.60^{*}$

$13.02 \pm$

$7.25^{\star}$

$0.001^{b}$

$8.63 \pm 6.44$

$8.54 \pm 6.28$

$7.83 \pm 6.26$

$7.98 \pm 6.71$

$0.877^{b}$

${ }^{*} \mathrm{p}<0,05$ versus $\mathrm{C} 1,{ }^{\mathrm{a}}$ Mann-Whitney Test; ${ }^{\mathrm{b}}$ Friedman/Dunn Test. 


\section{Cycle}
p-Value
$0.621^{\mathrm{a}}$
$0.001^{\mathrm{a}}$
$<0.001^{\text {a }}$
$0.001^{\mathrm{a}}$

${ }^{*} \mathrm{p}<0,05$ versus $\mathrm{C} 1,{ }^{a}$ Mann-Whitney Test; ${ }^{\mathrm{b}}$ Friedman/Dunn Test.

The mean salivary flow in the placebo group and PBMT groups showed no significant differences at any chemotherapy cycle and no significant variation throughout the study. The tooth mobility scores also showed no significant difference between the groups at any chemotherapy cycle. However, in the placebo group, there was a significant increase at $\mathrm{C} 3$ and $\mathrm{C} 4$ compared to $\mathrm{C} 1(\mathrm{p}=0.003)$, whereas in the PBMT group, there was no significant variation at any cycle $(p=0.534)($ Table 3$)$.

Both groups started chemotherapy treatment with equal mean OHIP-14 scores $(p=0.621)$. From C2 onwards, there was a significant increase in the dissatisfaction scores with oral health-related QoL in the placebo group $(p<0.001)$, remaining high compared to the PBMT group in C2 ( $p=0.001), C 3(p<0.001)$, and C4 $(p=0.001)$. The PBMT group showed no significant variation in OHIP-14 scores over the four cycles of chemotherapy $(p=0.877)$ (Table 3 ).

\section{PBMT prevents the reduction of objective taste loss in breast cancer patients treated with AC}

The objective evaluation of taste showed significant preservation of taste sensitivity in the PBMT group compared to the placebo group. All flavor sensitivity scores started with no difference between the two groups $(p>0.05)$. The sweet $(p=0.773)$, salt $(p=0.611)$, sour $(p=0.141)$ and bitter $(p=0.056)$ sensitivity showed no significant variation in the PBMT group (Supplementary File 2).

The sweet scores in the placebo group, there was a significant reduction at $\mathrm{C} 2(p<0.001)$, with a lower perception of sweet taste than the PBMT group at C2 $(p=0.001), C 3(p=0.009)$, and C4 $(p<0.001)$. Salty taste perception was significantly higher in the PBMT group at C2 $(p=0.010), C 3(p=0.001)$, and C4 ( $<$ $0.001)$. The perception of sour taste was significantly higher in the PBMT group at C2 $(p=0.006), C 3(p=$ $0.006)$, and C4 $(p=0.001)$. And at C3 $(p<0.001)$ and C4 $(p=0.001)$, bitter taste sensitivity was significantly higher in the PBMT group than in the placebo group (Supplementary File 2).

TSS of patients started with $54.39 \pm 21.77 \%$ taste perception in the placebo group and $57.08 \pm 21.20 \%$ in the PBMT group $(p=0.554)$. There was no change in the PBMT group throughout the study $(p=0.588)$, but the placebo group showed a significant reduction starting at $C 2(p<0.001)$. The objective taste perception scale showed significantly higher values in the PBMT group from C2 to C4 $(p<0.001)$ (Fig. 2).

\section{PBMT for prevention of chemotherapy-induced dysgeusia prevents the reduction of subjective taste loss in breast cancer patients treated with AC}

Subjective taste assessment showed similar onset between the two groups at $\mathrm{C} 1$ for both VAS $(\mathrm{p}=$ 0.313), CTCAE scale $(p=1.000)$, and STTA $(p=1.000)$. There was a significant increase in VAS 
dissatisfaction scores with taste $(\mathrm{p}<0.001)$ in both groups. However, while the PBMT group showed an increase from $\mathrm{C} 3$, the placebo group showed an increase from $\mathrm{C} 2$. VAS scores were also significantly higher in the placebo group than in the PBMT group at C2 $(p<0.001), C 3(p<0.001)$, and C4 $(p<0.001)$ (Fig. 2).

In both groups, CTCAE scores started at $0.00 \pm 0.00$ in both groups and increased significantly in the placebo group from $C 2(p<0.001)$ and in the PBMT group from C3 $(p<0.001)$. At C2 $(p<0.001), C 3(p<$ $0.001)$, and C4 ( $<0.001)$, the CTCAE dysgeusia scores were significantly higher in the placebo group than in the PBMT group. Taste STTA scores started at $0.00 \pm 0.00$ in both groups and increased significantly in the placebo group from $C 2(p<0.001)$ and in the PBMT group from C3 $(p<0.001)$. From C2 $(p<0.001), C 3(p<0.001)$, and C4 $(p<0.001)$, the STTA taste loss scores were significantly higher in the placebo group than in the PBMT group (Fig. 2).

\section{PBMT for prevention of dysgeusia secondarily reduces the incidence and severity of chemotherapy- related side effects in breast cancer patients treated with AC}

The PBMT-treated group showed a 0.43 -fold $(95 \mathrm{Cl}=0.35-0.53)$ reduction in the incidence of anorexia over the four cycles of chemotherapy $(p<0.001)$, and the incidence of diarrhea was 0.63 -fold $(95 \% \mathrm{Cl}=$ $0.41-0.97)$ lower in the PBMT group than in the chemotherapy protocol $(p=0.040)$. PBMT treatment did not significantly influence the incidence of nausea $(p=0.070)$; however, in the PBMT group, the incidence of oral mucositis was reduced $0.57(95 \% \mathrm{Cl}=0.36-0.92)$ times $(p=0.020)$, and the incidence of vomiting demonstrated a $0.75(95 \% \mathrm{Cl}=0.61-0.93)$ times reduction $(\mathrm{p}=0.008)$. Overall, the PBMT group showed a 0.89 -fold $(95 \% \mathrm{Cl}=0.85-0.93)$ reduction in the incidence of side effects related to the gastrointestinal tract $(\mathrm{p}<0.001)$ (Fig. 3).

\section{Discussion}

We showed that PBMT prevents dysgeusia during chemotherapy with AC. AC constitutes the first-line pharmacological treatment [4,23,24], but it is strongly related to side effects such dysgeusia [25]. Any disturbance in the ability to differentiate the perception of taste can modify salt and sugar intake, increase the risk of malnutrition, and affect the QoL $[25,26]$.

In a systematic review by Bressan et al. [27], it was observed that among the side effects caused by antineoplastic therapies, oral mucositis, nausea, vomiting, diarrhea, and dysgeusia were the most reported side effects associated with nutritional changes. Changes in taste due to chemotherapy may contribute to the high prevalence of malnutrition in cancer patients, and it is estimated that $50-70 \%$ of cancer patients suffer from taste disorders [5, 25].

The nutritional follow-up that most patients in this study underwent justifies the low weight loss in the placebo group, because most oncologic patients suffer from weight loss, causing a general worsening of their health status due to cachexia [27, 28], which leads to intolerance to therapy and a higher risk of other side effects $[10,29,30]$. Dysgeusia is a critical adverse effect in this process, interfering with the 
nutritional status $[5,8]$. In this study, PBMT lead to a significant increase in BMI, which may be associated with the low incidence of dysgeusia during treatment [30,31].

Taste is essential in generating satisfaction during food intake, and the reduction in gustatory function in breast cancer patients begins soon after the first cycle of chemotherapy [5]. The perception of salty taste has beenthe most affected [30]. This is a common side effect of systemic cancer therapy and negatively impacts the QoL of patients [32]. However, PBMT maintained taste quality and prevented loss of all taste scores.

Oppositely to PBMT, the placebo group showed an increase in the degree of tooth mobility. Dysentery is linked to side effects such as nausea and vomiting cravings $[8,29,31,33]$. The reduction of these side effects in the present study may have contributed to better oral hygiene and dental health status [34].

As previously described [29], gustatory function decreased throughout chemotherapy treatment regardless of the affected tastes, and the longer the treatment time, the higher the incidence of dysgeusia [35], directly affecting nutritional status [29]. The placebo group showed increasing objective (taste scores) and subjective (CTCAE, STTA, and VAS scores) alterations throughout the treatment for all the analyzed flavors from the second chemotherapy cycle, while in the PBMT group, these alterations were significantly controlled with a consequent reduction in the incidence of cachexia maintaining weight that is an important factor that contributes to general health status during cancer treatment.

In our study, besides controlling weight loss, general health status (ECOG) and anorexia, the proposed preventive PBMT protocol significantly controlled the increased incidence of nausea, vomiting, diarrhea, and oral mucositis [35-38]. The PBMT used in the present study as a preventive measure for dysgeusia played an essential role in preventing these various complications, which has already been described in literature [39-41].

Taste loss due to chemotherapy is strongly associated with inflammation and cytokines [42]. Because PBMT exhibits anti-inflammatory and antioxidant capacity [35, 36, 38, 43], we consider the mechanisms involved in preventing taste loss during chemotherapy with AC observed in this study to be locally inflammatory in nature [8].

The most significant limitation of the proposed protocol was the application time. Despite being extremely practical because it is a single application and can be performed on the day of the chemotherapy session, avoiding additional patient displacement (increased costs, discomfort, and dropout frequency), $[5,8]$ the application lasts 15-20 min, which tires the medical professional and the patient $[38,43]$. And, although this was a single-center study with a single chemotherapy protocol used, this is the first triple-blind randomized clinical trial that highlights the promising function of PBMT in preventing trans-chemotherapy taste loss.

\section{Conclusion}


PBMT is effective in preventing chemotherapy-induced dysgeusia and weight loss. PBMT reduced the incidence of gastrointestinal-related side effects and contributed to the maintenance of the general health status of breast cancer patients treated with AC.

\section{Declarations}

\section{ACKNOWLEDGMENTS}

We thank the Haroldo Juaçaba Hospital $(\mathrm{HHJ})$ - Ceará Cancer Institute for their contributions to this research, especially the SUS Chemotherapy service, for providing the infrastructure for the execution of this study.

\section{Funding}

Not applicable

\section{Conflicts of interest}

No conflicts of interest

\section{Ethics approval}

This study was approved by the Ethics Committee of the Haroldo Juaçaba Hospital (HHJ) (Instituto do Câncer do Ceará - ICC) under protocol number 3.286.363. All ethical aspects expressed in Resolution No. 466 of 2012 of the National Health Council/Ministry of Health, which brings the guidelines and regulatory standards for research with human beings and in accordance with the National Research Ethics Committee (CONEP) standard, were respected.

\section{Consent to participate}

Informed consent was obtained from all individual participants included in the study

\section{Consent for publication}

The authors affirm that human research participants provided informed consent for publication

\section{Availability of Data}

Not applicable

\section{Authors' Contribution}

All authors contributed to the study conception and design. Material preparation, data collection and analysis were performed by Cássia Emanuella Nóbrega Malta, Anna Clara Aragão Matos Carlos and Paulo Goberlânio de Barros Silva. The first draft of the manuscript was written by Cássia Emanuella 
Nóbrega Malta and Paulo Goberlânio de Barros Silva and all authors Manuele Carine Maciel de Alencar, Eveline Fernandes Alves e Silva,Victor Bruno Caitano Nogueira, Ana Paula Negreiros Nunes Alves, Fábio Figueiredo Chaves and José Fernando Bastos de Moura included patients, performed systemic analysis and commented on previous versions of the manuscript. All authors read and approved the final manuscript.

\section{References}

1. Gadisa DA, Assefa M, Wang SH (2020) Toxicity profile of doxorubicin-Cyclophosphamide and doxorubicin-Cyclophosphamide followed by Paclitaxel regimen and its associated factors among women with breast cancer in Ethiopia: A prospective cohort study. J Oncol Pharm Pract 26(8):19121920

2. Berruti A, Generali D, Kaufmann M, Puztai L, Curigliano G, Aglietta M (2011) International expert consensus on primary systemic therapy in the management of early breast cancer: highlights of the fourth symposium on primary systemic therapy in the management of operable breast cancer, Cremona, Italy. J Natl Cancer Inst Monogr 43(1):147-151

3. Harahap Y, Ardiningsih P, Winarti AC, Purwanto DJ (2020) Analysis of the Doxorubicin and Doxorubicinol in the Plasma of Breast Cancer Patients for Monitoring the Toxicity of Doxorubicin. Drug Des Devel Ther 25(14):3469-3475

4. Ongnok B, Chattipakorn N, Chattipakon SC (2020) Doxorubicin and cisplatin induced cognitive impairment: The possible mechanisms and interventions. Exp Neurol 32(4):113-118

5. Pugnaloni S, Vignini A, Borroni F, Sabbatinelli J, Alia S et al (2020) Modifications of taste sensitivity in cancer patients: a method for the evaluations of dysgeusia. Support Care Cancer 28(3):11731181

6. De Vries YC, Boesveldt S, Kelfkens CS, Posthuma EE (2018) Taste and smell perception and quality of life during and after systemic therapy for breast cancer. Breast Cancer Res Treat 170(1):27-34

7. De Vries YC, Van Den Ber MMGA, De Vries JHM, Boesveldt S (2017) Differences in dietary in take during chemotherapy in breast cancer patients compared to women without cancer. Support Care Cancer 25(8):2581-2591

8. PonticelliEM, Claris M, Frigerio M, Bergese I, Scavino E, Bernardini A (2017) Dysgeusia and healthrelated quality of life of cancer patients receiving chemotherapy: A cross-sectional study. Eur $\mathrm{J}$ Cancer Care 26(2)

9. Freitas PM, Simões A (2015) Lasers in dentistry: guideline for clinical practice. Willey Beckwell 49(1):27-33

10. Paglioni MP et al (2019) Tumor Safety and Side Effects of Photobiomodulation Therapy Used for Prevention and Management of Cancer Treatment Toxicities: A Systematic Review. Oral Oncol 93(1):21-28 
11. Zanin T, Zanin F, Carvalhosa AA, Souza PHC, Pacheco MT, Zanin ICJ, Brugnera A (2010) Use of 660 $\mathrm{nm}$ Diode Laser in the Prevention and Treatment of Human Oral Mucositis Induced by Radiotherapy and Chemotherapy.Photomedicine and. Laser Surgery 28(2):233-237

12. Mobadder ME, Farhat F, Mobadder WEM, Nammour S (2019) Photobiomodulation Therapy in the Treatment of Oral Mucositis, Dysphagia, Oral Dryness, Taste Alteration, and Burning Mouth Sensation Due to Cancer Therapy: A Case Series. Int J Environ Res Public Health 16(22):4505

13. De Oliveira RF, Silva AC, Simões A, Youssef MN, De Freitas PM (2015) Laser Therapy in the Treatment of Paresthesia: A Retrospective Study of 125 Clinical Cases. Photomedicine Laser Surgery 33(8):415-423

14. Zecha JAEM et al (2016) Low level laser therapy/photobiomodulation in the management of side effects of chemoradiation therapy in head and neck cancer: part 1: mechanisms of action, dosimetric, and safety considerations. Support Care Cancer 24(6):2781-2792

15. Löe H, Silness J (1963) Periodontal Disease in Pregnancy I. Prevalence and Severity. Acta Odontol Scand 21(6):533-551

16. Navazesh M (1993) Methods for collecting saliva. Ann NY Acad Sci 69(4):72-77

17. Albuquerque AFM, Soares ECS, de Barros Silva PG et al (2019) Clinical investigation of gustatory and neurosensory alterations following mandibular third molar surgery: an observational prospective study. Clin Oral Invest 23(1):2941-2949

18. National Cancer Institute (2017) Common Toxicity Criteria, Version 5.0 http://safetyprofilerctep.nci.nih.gov/CTC/CTC.aspx

19. Epstein JB, Barasch A (2010) Taste disorders in cancer patients: pathogenesis and approach to evaluation and treatment. Oral Oncol 46(2):77-81

20. Silveira MF, Marôco JP, Martins AMEBL, Marcopinto LF (2014) Impact of oral health on the physical and psychosocial dimensions: an analysis using structural equation modeling. Cad Saúde Pública 30(6):1-15

21. Oken M, Richard CH, Douglass CT, John MDH, Thomas ED, Eleanor ET, Paul PC (1982) Toxicity and response criteria of the Eastern Cooperative Oncology Group. American Journal Of Clinical Oncology 5(6):649-656

22. Sánchez-Lara K, Sosa-Sánchez R, Green-Renner D, Rodríguez C, Laviano A, Motola-Kuba D, Arrieta 0 (2010) Influence of taste disorders on dietary behaviors in cancer patients under chemotherapy. Nutr J 24(1):9-15

23. Yoshimoto $N(2020)$ Chemotherapy alters subjective senses of taste and smell but not dietary patterns in Japanese lung cancer patients. Support Care Cancer 28(4):1667-1674

24. Veronese P, Heachul DT, Scanavacca MI, Hajjar LA, Wu TC, Sacilotto L et al (2018) Effects of anthracycline, cyclophosphamide and taxane chemotherapy on CTc measurements in patients with breast cancer. PLoS One 13(5):763-784

25. Kuba S et al (2018) Awareness of Dysgeusia and Gustatory Tests in Patients Undergoing Chemotherapy for Breast Cancer. Support Care Cancer 26(11):3883-3889 
26. Turcott JG, Hernández EJ, Lara KS, Estrada DF, Barrón ZLZB (2020) Baseline Dysgeusia in Chemotherapy-Naïve Non-Small Cell Lung Cancer Patients: Association with Nutrition and QoL. Nutr Cancer 72(2):194-201

27. Bressan V, Stevanin S, Bianchi M, Aleo G, Bagnasco A, Sasso L (2016) The effects of swallowing disorders, dysgeusia, oral mucositis and xerostomia on nutritional status, oral intake and weight loss in head and neck cancer patients: A systematic review. Cancer Treat Rev 45(1):105-119

28. Álvaro Sanz E, Abilés J, Garrido Siles M, Pérez Ruíz E, Alcaide García J, Rueda D (2021) A Impact of weight loss on cancer patients' quality of life at the beginning of the chemotherapy. Support Care Cancer 29(2):627-634

29. Amézaga J, Alfaro B, Ríos Y, Larraioz A, Ugartemendia G (2018) Assessing taste and smell alterations in cancer patients undergoing chemotherapy according to treatment. Support Care Cancer 6(12):4077-4086

30. Boltong A, Keast R (2015) Chemosensory science in the context of cancer treatment: implications for patient care. Chemosens Percept 8(3):117-125

31. Tam SY, Tam VCW, Ramkumar S, Khaw ML, Law HKW, Lee SWY (2020) Review on the Cellular Mechanisms of Low-Level Laser Therapy Use in Oncology. Front Oncol 24(1):10:1255

32. Hovan AJ, Williams PM, Stevenson-Moore P, Wahlin YB (2010) A systematic review of dysgeusia induced by cancer therapies. Support Care Cancer 18(8):1081-1087

33. Martini S, lorio GC, Arcadipane F, Olivero F, Silvetti P, Rampino M, Garzino Demo P et al (2019) Prospective assessment of taste impairment and nausea during radiotherapy for head and neck cancer. Med Oncol 36(5):44

34. Yan R, Chen X, Gong X, Wang J, Yu J (2018) The association of tooth loss, tooth brushing, and QoL among cancer survivors. Cancer Med 7(12):6374-6384

35. Hong JH, Ozbek PO, Stanek BT, Dietrich AM, Duncan SE, Lee YW, Lesser G (2009) Taste and odor abnormalities in cancer patients. J Support Oncol 7(2):58-65

36. Zecha JAEM et al (2018) Low-Level Laser Therapy/photobiomodulation in the Management of Side Effects of Chemoradiation Therapy in Head and Neck Cancer: Part2: Proposed Applications and Treatment Protocols. Support Care Cancer 24(6):2793-2805

37. Elad S, Cheng KKF, Lalla RV, Yarom N, Hong C, Logan RM, Bowen J et al (2020) MASCC/ISOO clinical practice guidelines for the management of mucositis secondary to cancer therapy. Cancer126(19):4423-4431

38. Bensadoun RJ, Epstein JB, Nair RJ, Barasch A, Durlacher JER, Migliorati C (2020) Safety and efficacy of photobiomodulation therapy in oncology: A systematic review. Cancer Med 9(22):8279-8300

39. Nóbrega-Malta CE, Rodrigues MIQ, Lemos JVM, Duarte FB, Silva-Barros PG, Alves APNN (2020) Incidence and risk factors for oral mucositis after bone marrow transplantation patients under low intensity laser therapy: a longitudinal study. Journal of Bone Marrow Transplantation Cellular Therapy 2(1):1-11 
40. Oberoi S, Netto GZ, Beyene J, Treister N, Sung L (2014) Effect of Prophylactic Low Level Laser Therapy on Oral Mucositis: A Systematic Review and Meta-Analysis. PLoS One 9(9):e107418

41. Loncar B, Stipetic MM, Evic MB, Risovic D (2020) The effect of low-level laser therapy on salivary glands in patients with xerostomia. Photom Laser Surg 1:1-9

42. Feng P, Zhao H, Chai J, Huang L, Wang H (2012) Expression and secretion of TNF-a in mouse taste buds: a novel function of a specific subset of type II taste cells. PLoS One 7(8):e43140

43. Bitencourt GB, Motta LJ, Teixeira da Silva DF, Turcio KHL, Sfalcin RA, Gomes AMP, Mesquita-Ferrari RA, Fernandes KPS, Júnior AB, Bussadori SK (2021) Evaluation of the Preventive Effect of Photobiomodulation on Orofacial Discomfort in Dental Procedures: A Randomized-Controlled, Crossover Study and Clinical Trial. Photobiomodul Photomed Laser Surg 39(1):38-45

\section{Figures}




\section{CONSORT}

TRANSPARENT REPORTING of TRIALS

\section{CONSORT 2010 Flow Diagram}

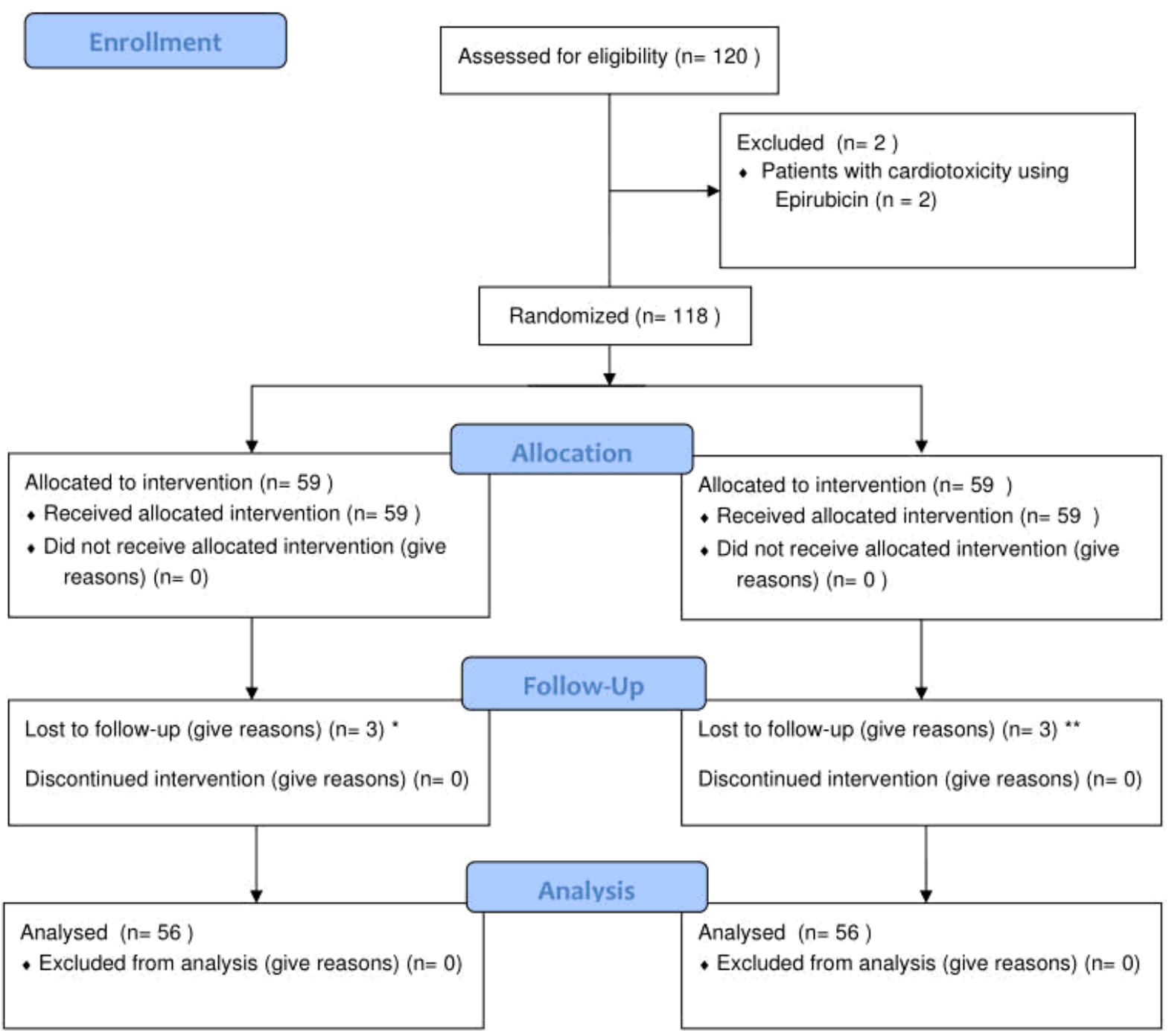

${ }^{*}$ Change of chemotherapy protocol $(n=2)$; Gave up on the long study $(n=1)$

**Chemotherapeutic toxicity $(n=2)$; Death $(n=1)$

\section{Figure 1}

CONSORT flowchart with inclusion, exclusion, and analysis criteria for breast cancer patients treated with Doxorubicin and cyclophosphamide and undergoing a randomized, triple-blind placebo-controlled clinical trial. 

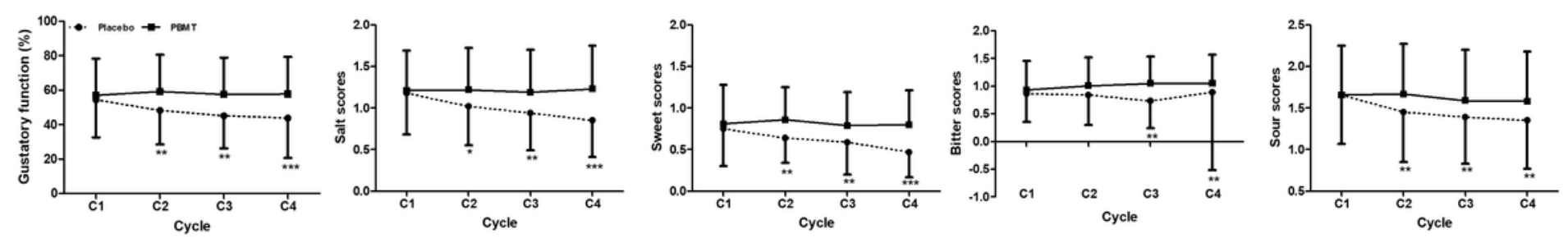

Figure 2

Objective and subjetive analysis of taste function over four administrations of Doxorubicin and cyclophosphamide in breast cancer patients undergoing PBMT protocol for dysgeusia prevention. 
$\begin{array}{llll}\text { PBMT } & \text { Placebo } & \text { Risk Ratio } & \text { Risk Ratio }\end{array}$

Events Total Events Total Weight IV, Fixed, $95 \% \mathrm{Cl}$ IV, Fixed, $95 \% \mathrm{Cl}$

Study or Subgroup Events Total Events Total Weight IV, Fixed, 95\% CI

1.2.2 Anorexia

C1

$\mathrm{C} 2$

$\mathrm{C} 3$

C4

Subtotal $(95 \% \mathrm{Cl})$

$\begin{array}{rr}17 & 56 \\ 17 & 56 \\ 17 & 56 \\ 19 & 56 \\ & 224\end{array}$

$40 \quad 56$

$1.3 \% \quad 0.42[0.28,0.65]$

$1.4 \% \quad 0.36[0.24,0.55]$

$\begin{array}{ll}1.4 \% & 0.40[0.26,0.62] \\ 1.4 \% & 0.56[0.37,0.85]\end{array}$

4256

Total events

70

$\begin{array}{rrrr}34 & 56 & 1.4 \% & 0.56[0.37,0.85] \\ & \mathbf{2 2 4} & \mathbf{5 . 5} \% & \mathbf{0 . 4 3}[0.35, \mathbf{0 . 5 3}]\end{array}$

Heterogeneity: $\mathrm{Chi}^{2}=2.24, \mathrm{df}=3(P=0.52) ; \mathrm{I}^{2}=0 \%$

Test for overall effect: $Z=7.81(P \leq 0.00001)$

\subsubsection{Diarrhea}

C1

$\mathrm{C} 2$

C3

C4

Subtotal $(95 \% \mathrm{Cl})$

Total events

$\begin{array}{rr}13 & 56 \\ 6 & 56 \\ 7 & 56 \\ 1 & 56 \\ & 224\end{array}$

$\begin{array}{rr}19 & 56 \\ 11 & 56 \\ 8 & 56 \\ 10 & 56 \\ & 224\end{array}$

$0.7 \% \quad 0.68[0.38,1.25]$

$0.3 \% \quad 0.55[0.22,1.37]$

$0.3 \% \quad 0.88[0.34,2.25]$

$0.1 \% \quad 0.10[0.01,0.76]$

$1.3 \% 0.63[0.41,0.97]$

Heterogeneity: $\mathrm{Ch}^{2}=3.81, \mathrm{df}=3(\mathrm{P}=0.28) ; \mathrm{I}^{2}=21 \%$

Test for overall effect: $Z=2.11(P=0.04)$

1.2.8 Nausea

$\begin{array}{lrrrrrr}\text { C1 } 1 & 54 & 56 & 56 & 56 & 66.7 \% & 0.96[0.91,1.02] \\ \text { C2 } & 47 & 56 & 53 & 56 & 14.4 \% & 0.89[0.78,1.01] \\ \text { C3 } & 36 & 56 & 37 & 56 & 3.4 \% & 0.97[0.74,1.28] \\ \text { C4 } & 30 & 56 & 30 & 56 & 2.1 \% & 1.00[0.71,1.41] \\ \text { Subtotal (95\% Cl) } & & \mathbf{2 2 4} & & \mathbf{2 2 4} & \mathbf{8 6 . 5 \%} & \mathbf{0 . 9 5}[\mathbf{0 . 9 0 , 1 . 0 0 ]}\end{array}$

Total events 167 176

Heterogeneity: $\mathrm{Chi}^{\mathbf{2}}=1.42, \mathrm{df}=3(\mathrm{P}=0.70) ; \mathrm{I}^{\mathbf{2}}=0 \%$

Test for overall effect: $Z=1.80(P=0.07)$

1.2.9 Oral mucositis

$\begin{array}{lrrrrrr}\text { C1 } & 7 & 56 & 7 & 56 & 0.3 \% & 1.00[0.38,2.66] \\ \text { C2 } & 3 & 56 & 9 & 56 & 0.2 \% & 0.33[0.10,1.17] \\ \text { C3 } & 4 & 56 & 16 & 56 & 0.2 \% & 0.25[0.09,0.70] \\ \text { C4 } & 10 & 56 & 13 & 56 & 0.5 \% & 0.77[0.37,1.61] \\ \text { Subtotal (95\% Cl) } & & 224 & & 224 & \mathbf{1 . 1 \%} & \mathbf{0 . 5 7}[\mathbf{0 . 3 6 , 0 . 9 2 ]} \\ \text { Total events } & 24 & & 45 & & & \end{array}$

Heterogeneity: $\mathrm{Ch}^{2}=5.06, \mathrm{df}=3(\mathrm{P}=0.17) ; \mathrm{I}^{2}=41 \%$

Test for overall effect: $Z=2.31(P=0.02)$

1.2.10 Vomiting

$\begin{array}{lrrrrrr}\text { C1 } & 33 & 56 & 41 & 56 & 3.4 \% & 0.80[0.61,1.05] \\ \text { C2 } & 16 & 56 & 27 & 56 & 1.0 \% & 0.59[0.36,0.97] \\ \text { C3 } & 14 & 56 & 17 & 56 & 0.7 \% & 0.82[0.45,1.50] \\ \text { C4 } & 10 & 56 & 15 & 56 & 0.5 \% & 0.67[0.33,1.35] \\ \text { Subtotal (95\% Cl) } & & 224 & & \mathbf{2 2 4} & \mathbf{5 . 5 \%} & \mathbf{0 . 7 5}[\mathbf{0 . 6 1 , 0 . 9 3 ]} \\ \text { Total events } & 73 & & 100 & & & \end{array}$

Heterogeneity: $\mathrm{Chi}^{2}=1.33, \mathrm{df}=3(\mathrm{P}=0.72) ; \mathrm{I}^{2}=0 \%$

Test for overall effect: $Z=2.66(P=0.008)$

$\begin{array}{lllll}\text { Total }(95 \% \mathrm{Cl}) & 1120 & 1120 & 100.0 \% & 0.89[0.85,0.93]\end{array}$

Total events 361 532

Heterogeneity: $\mathrm{Chi}^{2}=73.66, \mathrm{df}=19(\mathrm{P}<0.00001) ; \mathrm{I}^{2}=74 \%$

Test for overall effect: $Z=4.62(P \leq 0.00001)$

Test for subqroup differences: $\mathrm{Ch}^{2}=59.80, \mathrm{df}=4(\mathrm{P} \leq 0.00001),\left.\right|^{2}=93.3 \%$

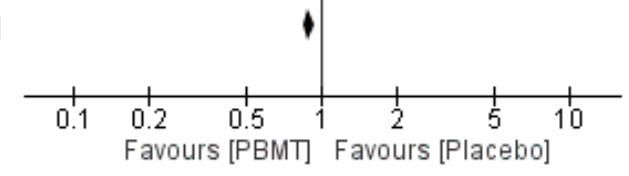

Figure 3

Valuation of incidence of chemotherapy-associated side effects over four sessions of AC administration

\section{Supplementary Files}

This is a list of supplementary files associated with this preprint. Click to download. 
- Supplementarfile01.tif

- Supplementarfile02.tif

- CONSORT2010Checklist.docx 